\title{
Wavelength-Selective Metamaterial Absorber for Thermal Detectors
}

\author{
A. Shoshi' ${ }^{1}$, W. Reichl' ${ }^{2}$, G. Niessner', T. Maier ${ }^{3}$, H. Brückl1 \\ ${ }^{1}$ Danube University Krems, Viktor Kaplan Strasse 2, A-2700 Wr. Neustadt, Austria \\ astrit.shoshi@donau-uni.ac.at \\ 2 E+E Elektronik GmbH, Langwiesen 7, A-4209 Engerwitzdorf, Austria \\ ${ }^{3}$ Austrian Institute of Technology GmbH, Donau-City-Strasse 1, A-1220 Vienna, Austria
}

\begin{abstract}
The absorption properties of trilayer and more sophisticated multilayer metamaterial structures are analyzed by numerical simulations. The dissipative electromagnetic energy absorption of tailored metamaterials can be utilized to improve the performance of thermal detectors. The absorption properties such as absorption resonance frequency, amplitude and spectral bandwidth depend on the geometry of the metamaterials. Here, we consider isotropic disk-shaped components of Al- and Al/AINstacked absorber elements. They show spectral resonances at wavelengths between 2 and $6 \mu \mathrm{m}$, reaching near unity absorption with narrow bandwidths ranging from $0.45 \mu \mathrm{m}$ to $1.05 \mu \mathrm{m}$. Multilayered metamaterial structures show multiband perfect absorption which are for simultaneous multispectral infrared imaging.
\end{abstract}

Key words: Metamaterials, Perfect Absorber, Thermal Detector and Imaging, Wavelength-Selective, Integrated Sensor

\section{Introduction}

Metamaterials are artificial compositional structures which exhibit physical properties different from the intrinsic properties of the individual material components [1]. Optical properties such as the negative refractive index [2] and electromagnetic cloaking [3] can be observed, which are not accessible in naturally occurring materials. In general, periodically arranged metal-dielectric structures with unit cell dimensions in the sub-wavelength regime are employed to independently tune the electric [4] and magnetic [5] resonances evoked by the incident radiation. The circulating surface currents induced by plasmon resonance in the metallic layer accompanied by the displacement field in the dielectric layer [6] can be manipulated by changing the geometrical and material parameters. Metamaterials can be characterized by the complex effective electric permittivity $\varepsilon(\omega)=\varepsilon_{1}+i \varepsilon_{2}$ and magnetic permeability $\mu(\omega)=\mu_{1}+i \mu_{2}$ [7]. In research, much attention has been paid to the real part of $\varepsilon$ and $\mu$ to create materials with negative refractive index, while at the same time minimizing the undesired losses. Similarly, the imaginary loss terms $\varepsilon_{2}$ and $\mu_{2}$ can be engineered to achieve high attenuation and consequently large absorption. By independently manipulating resonances in $\varepsilon$ and $\mu$, it is possible to effectively absorb both the incident electric and magnetic field. Moreover, the impedance of the metamaterial, $Z=\sqrt{\varepsilon(\omega) / \mu(\omega)}$, can be matched to free space giving rise to minimized reflectivity [8]. The perfect metamaterial absorber (PMA) is defined to have absorption near unity.

The enhanced absorption properties of metamaterial can be utilized to tailor the spectral responsivity of thermal sensors [8-10]. Thermal sensors, like bolometer, thermopile, and pyroelectric sensor, convert temperature changes caused by the absorption of incident light into an electrical signal. They usually have a broad spectral response. Exchangeable or fixed optical filter units are used for wavelength selection. In order to achieve a large sensor responsivity, a large absorption is required. Therefore, metamaterials directly integrated on top of thermal sensors are wavelength-selective with an effective absorption and heat transfer [9, 10].

This paper aims towards the understanding of the properties and composition of micron-sized metamaterial stacks and their utilization as wavelength-selective PMA in thermal sensors.

\section{Materials and Simulation Model}

As composite dielectric/metallic materials, highly conductive Aluminum (Al) with low ohmic losses 
and non-dissipative Aluminum nitride (AIN) dielectric interlayer were chosen. Al is a good thermal and electrical conductor. AIN has superior thermal and mechanical properties in the infrared. In particular, the high thermal conductivity of AIN is comparable to metals such as $\mathrm{Al}$ and is about ten times higher compared to Al oxide [11]. This can be beneficial for the heat transfer to the energy transducer in an integrated sensor. Moreover, these materials are compatible with standard microelectronic production processes

A simple layout of a near unity absorber mitigates lithographic demands (Fig.1). The disc-like top metallic layer (resonator) of an Al/AIN/Al-trilayer is located in the center of the square-shaped unit cell consisting of the bottom metal and the dielectric layer of side length $p$. Due to the circular resonator shape, the excitation of the resonance is expected to be isotropic and independent on radiation polarization [6]. As will be discussed later, the trilayer absorber is additionally extended to a multilayer absorber with alternating dielectric/metallic stacks (Fig. 1). Moreover, a passivation layer, which is usually found in thermal sensors, e.g. $\mathrm{SiO}_{2}$ in thermopiles, $\mathrm{Si}_{3} \mathrm{~N}_{\mathrm{x}}$ in bolometers or $\mathrm{Au}$ in pyroelectric detectors, is also considered in the layout (Fig. 1). The areal density of the metamaterial is determined by the disk radius in the unit cell.

Finite-element simulations based on the commercially available software package COMSOL Multiphysics were performed to analyze the optical properties of a reduced threedimensional model (Fig. 1). The incident light is an electromagnetic plane wave propagating at normal incidence along the negative z-direction. The boundary conditions for the unit cell outer walls perpendicular and parallel to the electric field are perfect electric and perfect magnetic conductor, respectively. For the top and bottom outer walls scattering boundary conditions with and without an incident wave are used, respectively. The experimental refractive index values of the individual materials including the dispersion relation are taken from [12-14] and implemented in the model. The field distributions and the time averaged power flow in the structure is calculated. Reflection is determined by simulating the same elementary cell geometry with all layer domain properties set to vacuum and normalized to the incident intensity. The difference in the integrated power flow at the individual layer boundaries reveal the absorption values as $A(\omega)=1-R(\omega)-T(\omega)$, with the frequency dependent reflectance $R(\omega)$ and transmittance $T(\omega)$.

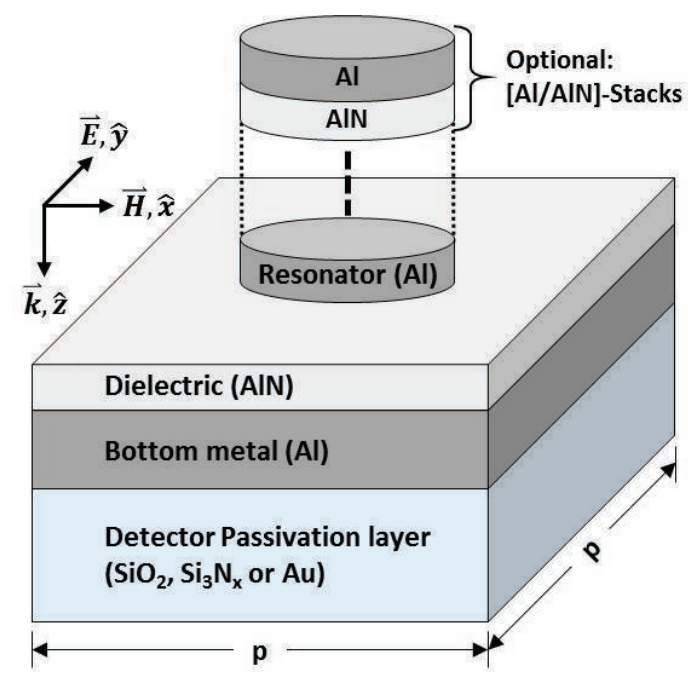

Fig. 1. Schematic illustration of the unit cell in the numerical simulations.

\section{Electric and Magnetic Resonances}

The incident electromagnetic plane wave propagating in negative z-direction excites plasmon resonances in the top metallic layer and, thus, polarization currents depending on the electrical permittivity appear inside the metal. The resonator layer at the top behaves like an electric dipole that serves as a coupler to the electric field of the incident wave. Consequently, the electromagnetic fields are concentrated within sub-wavelength regimes leading to a significant increase of the local field strengths (Fig. 2, a)). The origins of magnetic resonances are anti-parallel currents in the metallic layers, which, together with the displacement field in the dielectric layer, result in circulating currents [6, 15]. The current loop induces a magnetic dipole moment that can resonantly couple to the magnetic field vector of the incident light. For a strong localization of the electromagnetic energy within the metamaterial, both, an electric and a magnetic dipole resonant coupling at the same frequency is necessary. This resonant coupling is of destructive nature for the reflected direction, thus eliminating reflection. In a PMA, neither reflections nor transmissions of the incident light can be observed $[5,16]$.

The simulated electromagnetic field distributions within the absorber element where both resonances appear are exemplarily shown in Fig. 2. Here, typical geometric parameters for resonator radius and thickness, dielectric, bottom metal and SiO2-substrate thicknesses are $500 \mathrm{~nm}, 50 \mathrm{~nm}, 110 \mathrm{~nm}, 150 \mathrm{~nm}$ and 250 $\mathrm{nm}$, respectively. The distribution of the electric field in Fig. 2 a) shows the typical dipole excitation and spatial localization within the dielectric interlayer. The concentration of the magnetic field in the dielectric layer demonstrates the confinement of the magnetic 
field caused by the oscillating current loop (Fig. $2 \mathrm{~b})$ ). The inhomogeneous distribution of the current density in both metal layers is depicted in Fig. 2 c). For smaller wavelengths far from resonance, the surface current density displays a rather homogeneous distribution with significantly lower current amplitudes. Approaching the resonance wavelength, the current density experiences a progressive concentration at the center of the resonator and reaches its maximum value at resonance wavelengths. For wavelengths beyond the resonance, the current density steadily decreases. Noteworthy is that the surface current reverses its sign from positive to negative resulting in a directional change of the circulating current loop. The normalized current density magnitude is slightly asymmetric for wave-lengths smaller and larger than the resonance wavelength. The induced image charges in the bottom metal layer behave similar to those observed in the top metal layer. The current sheets in the bottom layer are antiparallel oriented and show slightly lower amplitudes compared to the top layer. In agreement to theoretical predictions, the current sheets in both metallic layers form a circulating current loop, have comparable amplitudes, and are slightly out of phase [6, 16]. Figure 2 d) displays the according time averaged energy flow of the electromagnetic wave in the absorber element at resonance.

\section{Simulation Results: Geometry Variations and Absorption}

The influence of all geometrical parameters on the absorption behavior of metamaterials was analyzed. Except for the multilayer systems, all results presented here correspond to trilayer absorbers.

Fig. 3 a) shows a selection of absorption spectra for resonator radii varying between 200 and $600 \mathrm{~nm}$, while all other parameters were kept constant. The layer thicknesses of the resonator, dielectric, bottom metal, and substrate are $50 \mathrm{~nm}, 115 \mathrm{~nm}, 50 \mathrm{~nm}, 250 \mathrm{~nm}$, respectively. The unit cell side length was $2.1 \mu \mathrm{m}$. A variation of the resonator radius results in a clear shift of the resonance peak which indicates a change in the effective dielectric permittivity of the metamaterial structure. The resonance wavelength experiences a linear red shift with increasing radius. According to a linear fit, a radius change of $10 \mathrm{~nm}$ causes a shift in the resonance wavelength of $80 \mathrm{~nm}$. This is a good reference value in order to estimate peak shifts due to geometric tolerances caused by fabrication errors. The full width half maximum (FWHM) of the absorption peak gives information about the coupling strength of excited oscillations in the metamaterial system. There is a linear depend-
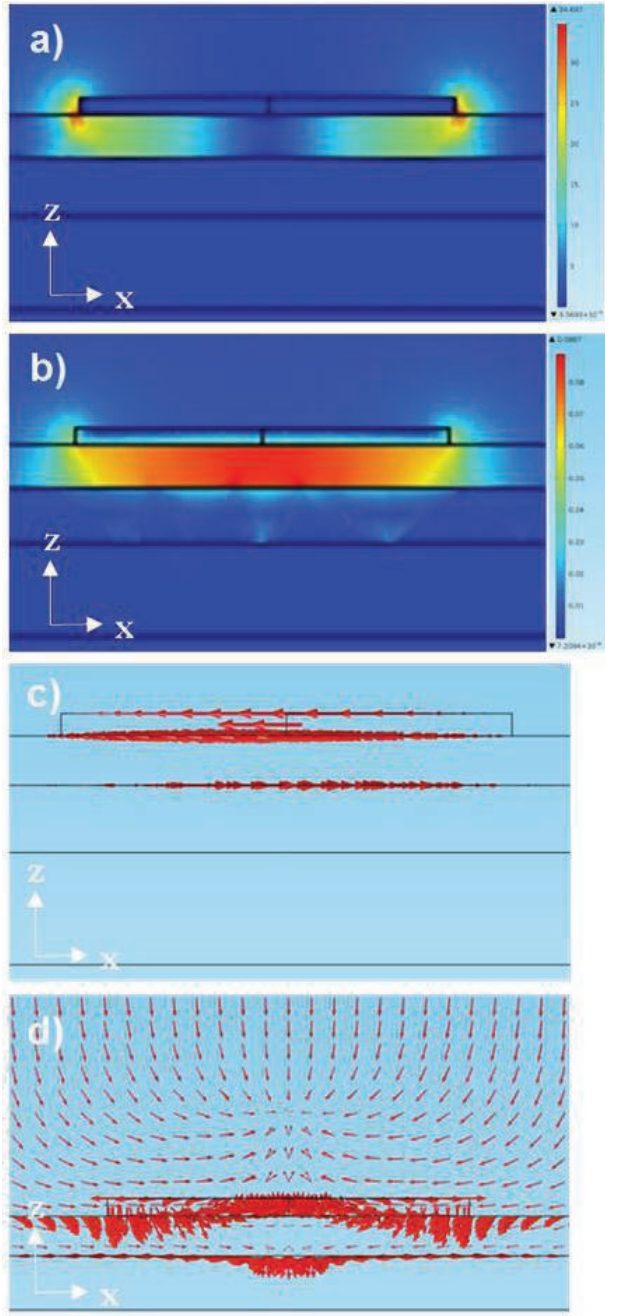

Fig. 2. Optical properties of metamaterials for simultaneous electric and magnetic resonances. a) Normalized electric field and b) magnetic field. c) Surface current density. d) Time averaged energy flow defined by the Poynting vector.

ency on the resonator radius ranging from 200 to $600 \mathrm{~nm}$ with FWHM values of 100 and $700 \mathrm{~nm}$, respectively.

Fig. 3 b) presents the calculated absorption properties for varying dielectric thicknesses between 10 and $210 \mathrm{~nm}$, while all other parameters remain constant: resonator radius and height, bottom metal and substrate thicknesses, and unit cell length are $525 \mathrm{~nm}$ and $50 \mathrm{~nm}, 50 \mathrm{~nm}$ and $250 \mathrm{~nm}$, and 2,1 $\mu \mathrm{m}$, respectively. A change in the dielectric thickness influences primarily the inductance or magnetic resonance, and thus, the effective magnetic permeability of the absorber system. A varying dielectric thickness also affects the capacitive coupling to the bottom metal layer, i.e. it changes the effective permittivity, but in a less pronounced manner. Basically, lower layer thicknesses result in higher capacity and lower inductance of the system and vice versa. The resonance frequency remains constant at a 
resonance wavelength of $4.5 \mu \mathrm{m}$ in a broad thickness range of $70-210 \mathrm{~nm}$ (Fig. $3 \mathrm{~b}$ )). This behaviour reflects the relation of the resonance frequency $\left(\omega_{0}\right)$ to the capacitance (C) and inductance $(L)$ of the structure, which can be considered as an undamped resonant circuit:

$\omega_{0}=1 / \sqrt{L C}$

For dielectric thicknesses below $70 \mathrm{~nm}$, there is a significant red shift of the resonance along with a reduction in the amplitude, which is characteristic for a damped resonance. At this dimensions, the size effect becomes more important, and an additional inertial inductance $\left(L_{i}\right)$ occurs [17]. The contribution of the inertial inductance and the geometrical inductance $\left(L_{g}\right)$ to the total inductance $\left(L=L_{g}+L_{i}\right)$ of the structure are related to the layer thickness and determine the damping of the resonance. Due to the additional losses, the magnetic resonance frequency does not solely scale up with the geometrical inductance $\left(L_{g}\right)$. By introducing an effective damping factor to eg. (1), both, a shift of the resonance frequency and a reduction of the amplitude can be explained. The calculations show, that the amplitude reaches its maximum value of 0.96 at a dielectric thickness of $110 \mathrm{~nm}$ and decreases for larger and smaller layer thickness values similar to a negative parabolic function. The absorption remains within the range $90-130 \mathrm{~nm}$ around 0.9 . The FWHM value of about $475 \mathrm{~nm}$ is lowest at a thickness of $110 \mathrm{~nm}$ and it remains within the range of 90 $130 \mathrm{~nm}$ almost constant. Smaller or larger layer thicknesses result in a FWHM increase up to $950 \mathrm{~nm}$.

In a PMA, the layer thickness of both the resonator and the bottom metal needs optimization. The penetration depth of an electromagnetic wave into a metallic layer and, thus, the transmission $T(\omega)$, is determined by the wavelength dependent skin effect. If the thickness of the bottom layer is smaller than the skin depth, image charges are not effectively formed in the bottom metal layer, deteriorating the magnetic resonance. This also implicates a dielectric dipole resonance without a proper impedance matching. Therefore, an effective energy transfer is hindered and unwanted reflections might occur [6]. The resonator (bottom layer) thickness range of 30 to $150 \mathrm{~nm}$ (30 to $200 \mathrm{~nm}$ ) was analyzed. The results shown in Fig. $3 \mathrm{c}$ ) are $525 \mathrm{~nm}$ radius, $110 \mathrm{~nm}$ dielectric height, $250 \mathrm{~nm}$ substrate height and $2.1 \mu \mathrm{m}$ unit cell length. For all investigated thickness permutations, the absorption remains always larger than 0.9 and reaches the near unity absorption value of 0.995 for the pair combination of $50 \mathrm{~nm}$ resonator thickness and $150 \mathrm{~nm}$ bottom metal thickness.
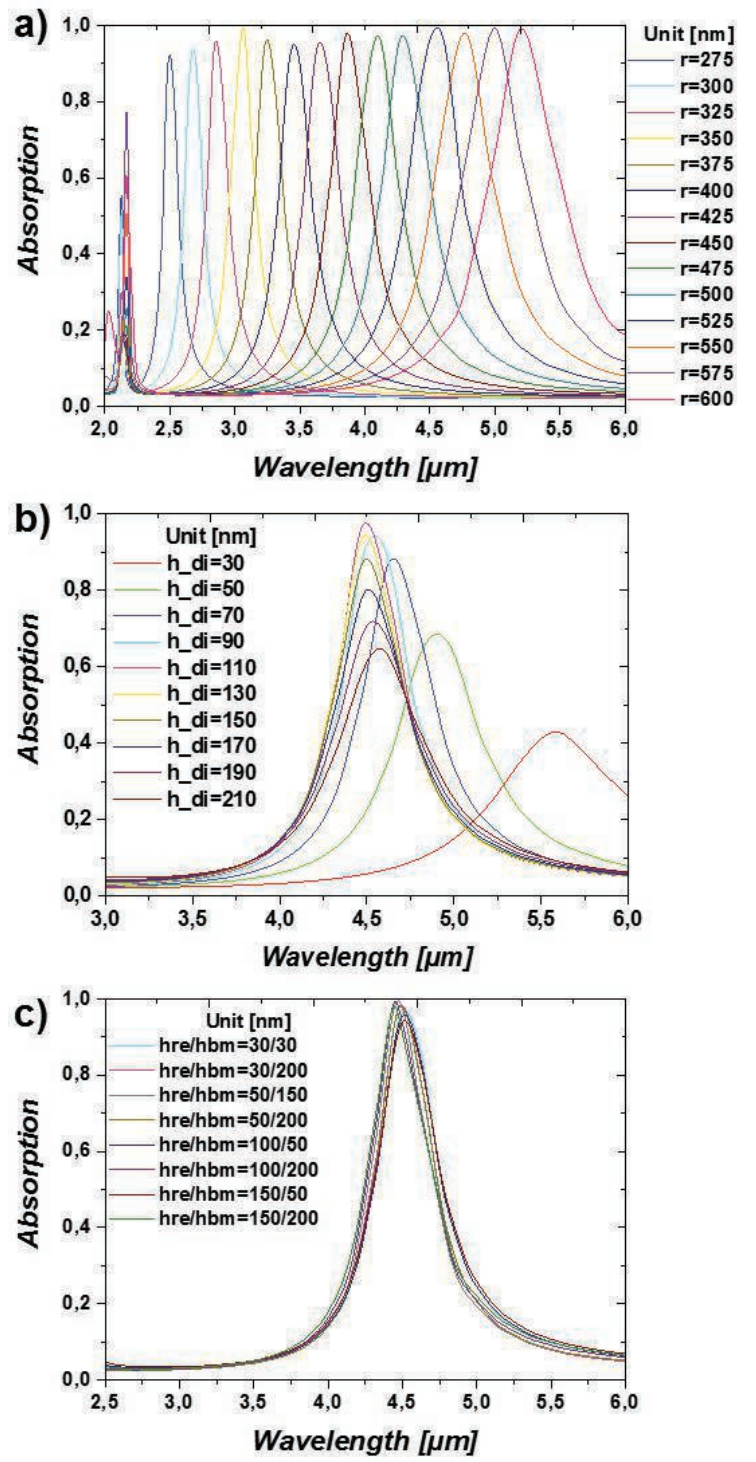

Fig. 3. Numerical calculations of absorption spectra for varying a) resonator radius, b) dielectric layer thickness and c) resonator (hre) and bottom metal (hbm) thickness

The position of the resonance peak remains constant for all combinations. The FWHM has a slightly oscillatory behavior within the range of $495 \pm 25 \mathrm{~nm}$.

Thermal detectors are capped e.g. by insulating layers such as $\mathrm{SiO}_{2}, \mathrm{Si}_{3} \mathrm{~N}_{x}$ or conductive $\mathrm{Au}$ layers. Their influence on the absorption of integrated metamaterials is also investigated in the simulation model (Fig. 1). In all simulations up to here, a $\mathrm{SiO}_{2}$-substrate of $250 \mathrm{~nm}$ was assumed. $\mathrm{SiO}_{2}$-thickness variations up to $3 \mu \mathrm{m}$ show no noticeable changes in the absorption behavior (data not shown). In the case of a gold passivation layer variation (30 to $200 \mathrm{~nm}$ ), a slightly decreased absorption within the range of 50 and $110 \mathrm{~nm}$ from near unity to 0.95 could be observed. Since the transmission $T(\omega)$ in this metamaterial structure is negligible, the marginal 

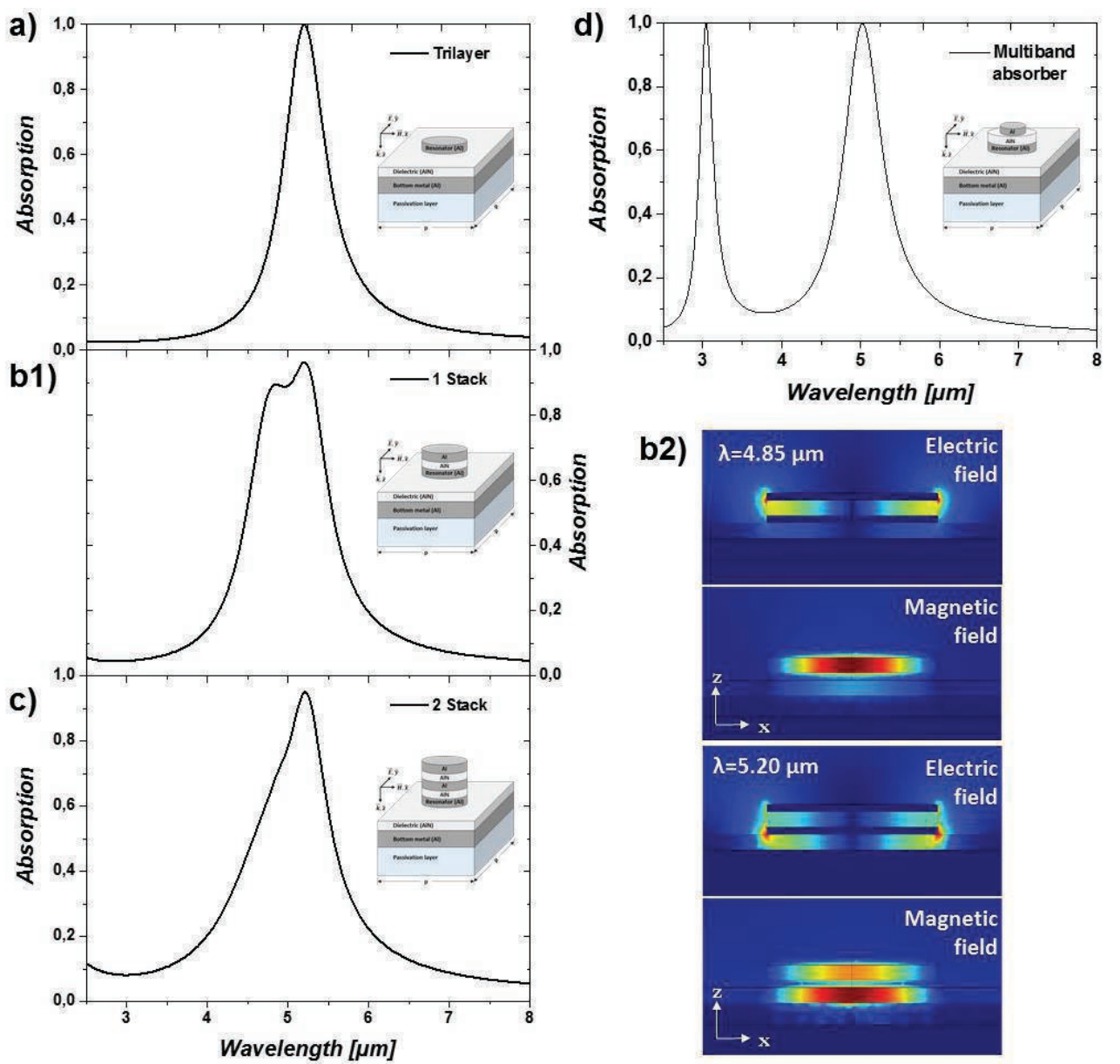

b2)

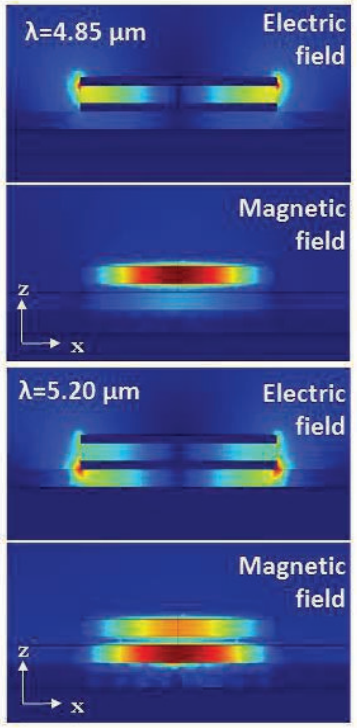

Fig. 4. Multilayer absorption spectra for a a) trilayer, b) 1-stack and c) 2-stack system. b2) Electric and magnetic field in the absorber at different resonance wavelengths related to absorption spectra shown in b1). d) Multiband absorber based on a modified 1-stack absorber. The insets show the specific stack configurations

absorption deviations can be explained by changes in the image charge formation in the bottom layer and energy flow direction affected by the conductive Au layer.

The areal density, i.e. resonator base surface area compared to the elementary cell base surface area, affects the absorption. An areal density of more than $50 \%$ is required for high absorption values, while a decreasing absorption is observed for smaller values (data not shown). Similar to previous studies [9, 10], there is no indication of coupling between the absorber elements down to a lateral absorber-toabsorber distance of $600 \mathrm{~nm}$. One reason could be the short-range nature of the concentrated field distribution along the $x$-axis at resonant frequency (Fig. 2 a) \& b)). Coupling phenomena occur at smaller distances. This is demonstrated in Fig. 4. Coupling phenomena appear in multilayer systems if additional dielectric/metallic layers are stacked on top of the resonator disc. These disc-shaped, alternating dielectricmetallic layers have thicknesses of 50/100 nm, respectively. Note that an $n$-stack system represents the trilayer system with $n$ additional
AIN/Al-stacks on top of the resonator. The following simulation parameters remain constant during the calculations: resonator radius and height, dielectric, bottom metal and $\mathrm{SiO}_{2}-$ substrate height are $500 \mathrm{~nm}$ and $50 \mathrm{~nm}, 115 \mathrm{~nm}$, $150 \mathrm{~nm}, 250 \mathrm{~nm}$, respectively. The absorption spectrum of a 0 -stack system in Fig. 4 a) corresponds to the trilayer system at resonance frequency and serves here as a reference. Characteristic for the absorption behavior of a 1-stack system is a spectral peak broadening. This peak broadening from $650 \mathrm{~nm}$ to $1050 \mathrm{~nm}$ is attributed to the excitations of a second resonance at $4.85 \mu \mathrm{m}$ and its superposition with the absorption peak at $5.2 \mu \mathrm{m}$ of the trilayer (Fig. 4 b1)). The absorption peak at $4.85 \mu \mathrm{m}$ results from a resonant coupling of the incident light solely in the upper stack, which is indicated by the concentration of the electric and magnetic field in the upper circular dielectric layer (Fig. 4 b2)). At the wavelength of $5.2 \mu \mathrm{m}$ both fields are dominantly localized in the dielectric of the trilayer. The difference in the excitation frequency arises from lateral geometrical differences. The reduced metallic area in the first 
stack might result in confined surface currents leading to two different effective permittivities and permeabilities. In a 2-stack system both, the electric and magnetic fields are concentrated in the dielectric layers of the first two stacks (Fig. 4 b2)). Since the two stacks hold the same geometry, the two resonances seem to merge to only one absorption peak, while the spectral broadening remains the same. Simulation results for 3-stack systems revealed similar absorption properties.

A modified design of the shown 1-stack system was realized as a wavelength selective "perfect multiband absorber" (Fig. 4d)). The tailored radius of the top disc is $350 \mathrm{~nm}$, while the radius of the underlying dielectric and metallic layer is $575 \mathrm{~nm}$. Except of the radii, all other parameters remain identical. In accordance to Fig. 2 a), two absorption peaks appear at a resonance wavelength of $3.0 \mu \mathrm{m}$ and $5.0 \mu \mathrm{m}$ for absorber radius $350 \mathrm{~nm}$ and $575 \mathrm{~nm}$, respectively, although the disks are stacked in this case. The absorption for both spectral bands reaches values very close to unity (>99,9\%). The electric and magnetic resonance properties display similar characteristics as the unmodified 0 -stack metamaterial structure.

\section{Conclusion}

Micron-sized composite structures consisting of aluminum and aluminum nitride layer stacks have been investigated as potential wavelengthselective absorbers for thermal detectors by numerical simulations. Electric and magnetic resonances are optimized by tuning the geometrical parameters of the absorber elements, resulting in near unity absorption. The circularly patterned resonator structures implicate polarization-independent absorption properties. The integration of metamaterial structures in thermal detectors has been addressed by the implementation of typical passivation layers in the simulation model. No significant influences on the absorption properties could be observed. More complex metamaterials consisting of patterned multilayer resonators show a spectral broadening of the absorption peak due to additional resonances in close proximity to each other. Multilayer structures of different sized resonators were designed, which can be utilized as multiband PMA. Experimental realization and spectroscopic characterization of the presented metamaterial structures are part of current investigations.

\section{References}

[1] V. G. Veselago, Soviet Physics Uspekhi 10, 509 (1968); doi:10.1070/PU1968v010n04ABEH003699
[2] R. A. Shelby, D. Smith, and S. Schultz, Science 292, 77-79 (2001); doi: 0.1126/science. 1058847

[3] D. Schurig, J.J. Justice, S.A. Cummer, J.B. Pendry, A.F. Starr, and D.R. Smith, Science 314, 977-980 (2006); doi: 10.1126/science.1133628

[4] J.B. Pendry, A.J. Holden, W.J. Stewart, and I. Youngs, Physical Review Letters 76, 4773-4776 (1996); doi: http://dx.doi.org/10.1103/PhysRevLett.76.4773

[5] J.B. Pendry, A.J. Holden, D.J. Robbins, and W. J. Stewart, IEEE Transactions on Microwave Theory Techniques 47, 2075-2084 (1999); doi: $10.1109 / 22.798002$

[6] G. Dayal, and S. A. Ramakrishna, Optics Express 20, 17503-17508 (2012); doi: http://dx.doi.org/10.1364/OE.20.017503

[7] D.R. Smith and J.B. Pendry, Journal of the Optical Society of America B 23, 391-403 (2006); doi: http://dx.doi.org/10.1364/JOSAB.23.000391

[8] N. I. Landy, S. Sajuyigbe, J. J. Mock, D.R. Smith, and W. J. Padilla, Physical Review Letters 100, 207402 (2008); doi: http://dx.doi.org/10.1103/PhysRevLett.100.20740 2

[9] T. Maier, and H. Brueckl, Optics Letters 34, 30123014 (2009); doi: http://dx.doi.org/10.1364/OL.34.003012

[10] T. Maier, and H. Brueckl, Optics Letters 35, 37663768 (2010); doi: http://dx.doi.org/10.1364/OL.35.003766

[11] Y. Zhao, C. Zhu, S. Wang, J. Z. Tian, D. J. Yang, C. K. Chen, $\mathrm{H}$. Cheng, and P. Hing, Journal of Applied Physics 96, 4563-4568 (2004); doi: http://dx.doi.org/+10.1063/1.1785850

[12] J. Kischkat, S. Peters, B. Gruska, M. Semtsiv, M. Chashnikova, M. Klinkmüller, O. Fedosenko, S. Machulik, A. Aleksandrova, G. Monastyrskyi, Y. Flores, and W. T. Masselink, Applied Optics 51, 6789-6798 (2012); doi: 10.1364/AO.51.006789.

[13] M. A. Ordal, R. J. Bell, R. W. Alexander, Jr., L. L. Long, and M. R. Querry, Applied Optics 24, 44934499 (1985); http://dx.doi.org/10.1364/AO.24.004493

[14] E. D. Palik, Handbook of Optical Constants of Solids (Academic, New York, 1985).

[15] H. Tao, N. I. Landy, C. M. Bingham, X. Zhang, R. D. Averitt, and W. J. Padilla, Optics Express 16, 7181-7188 (2008); http://dx.doi.org/10.1364/OE.16.007181

[16] Y. Zeng, H.-T. Chen, and D. A. R. Dalvit, Optics Express 21, 3540-3546 (2013); http://dx.doi.org/10.1364/OE.22.006519

[17] S. O'Brien, and J.B. Pendry, Journal of Physics: Condensed Matter 14, 6383-6394 (2002); doi:10.1088/0953-8984/14/25/307 